\title{
Blue Light Emitting Silicon Nanocrystals Prepared by Laser Ablation of Doped Si Wafers in Water
}

\author{
Vladimir SVRCEK and Michio KONDO

\begin{abstract}
Novel Si Material Team, Research Center for Photovoltaics, National Institute of Advanced Industrial Science and Technology (AIST), Central 2, Umezono 1-1-1, Tsukuba, 305-8568, JAPAN

E-mail:vladimir.svrcek@aist.go.jp
\end{abstract}

\begin{abstract}
The water-confinement of nanosecond-pulsed laser generated plasma provides great potential for preparation of ambient-stable silicon nanocrystals ( $\mathrm{Si}$-ncs) with quantum confinement size effect. Here, we report on blue-luminescent Si-ncs prepared by nanosecond $\mathrm{KrF}$ excimer laser ablation of doped-crystalline silicon target immersed in water. The Photoluminescence (PL) at low temperature show typical red-shift of the maxima assigned to the quantum confinement effect in Si-ncs. An introduction of ambient stable and less luminescent p-type doped Si-ncs into conjugated (poly(3hexylthiophene) (P3HT) polymer leads to an interaction between electronic states of the nanocrystal and the polymer. An exciton transfer from the polymer into the nanocrysatal is observed. At the same time Si-ncs blended with P3HT polymer form a bulk-heterojunction. Dissociation of the exciton and the photoconductivity generation under 1.5AM irradiation is evidenced. The ratio value of $\sim 1.7$ between photo- and dark-conductivity is achieved at $\sim 55 \mathrm{wt}$. \% concentration of Si-ncs in P3HT polymer.

DOI:10.2961/jlmn.2010.02.0001
\end{abstract}

Keywords: nanosecond-pulsed laser ablation, silicon nanocrystals, photoluminescence, bulkheterojunction

\section{Introduction}

Laser ablation synthesis of different kinds of nanoparticles with peculiar electrical and optical properties has attracted great interest in many research fields in last decade [1-3]. Silicon nanocrystals (Si-ncs) represent an important type of attractive nanoparticles due to the mature of silicon technologies and potential applications [4, 6-8]. Recently a synthesis of luminescent Si-ncs by cost effective nanosecond laser ablation process in liquid media was reported [9, 10]. The confinement of nanosecond-pulsed laser generated plasma in water offers stable surface and allows formation of room temperature luminescent Si-ncs with quantum confinement size effect $[11,12]$.

Since the synthesis of air-stable-blue PL from Si-ncs at room temperature has still remained exclusive such costeffective approach can considerably impact development of optoelectronic devices. It is believed that the PL from Sincs with wide energy $(>2 \mathrm{eV})$ can bring faster operation of potential devices. On the other hand it is expected that nanocrystal doping might impact the PL performance. Functionalization of the doped-luminescent Si-ncs with polymers might lead to a fabrication of new class of nanomaterials with peculiar properties [13]. Particularly, conductive conjugated polymers let simple integration of the colloidal nanoparticles and might allow design of nanocompositematerials for optoelectronic or photovoltaics at low cost [14].

In this paper the nanocrystals by relatively cheap and scalable method are prepared. A nanosecond excimer pulsed laser is employed for formation of blue-luminescent Si-ncs in water. The silicon boron and phosphorus doped silicon crystalline wafers and immersed in water are used for synthesis. An introduction of ambient stable and less luminescent boron-doped Si-ncs into conjugated (poly(3hexylthiophene) (P3HT) polymer is demonstrated. Electronic interaction between the polymer and the nanocrystal results an exciton transfer from the polymer to the Si-ncs through a bulk-heterojunction formation.

\section{Experimental details}

In this work we follow the synthesis route of blue luminescent Si-ncs based on a recently developed waterconfined nanosecond laser ablation process [9, 12]. Particularly, the Si-ncs are prepared by nanosecond excimer pulsed laser $\left(\mathrm{KrF}, 245 \mathrm{~nm} 20 \mathrm{~Hz}, 20 \mathrm{~ns}, \sim 20 \mathrm{~mJ} / \mathrm{cm}^{2}\right)$. Crystalline silicon boron (p-type, $<100>$, concentration of $\sim 3 \times 10^{16} \mathrm{~cm}^{-3}$ ) and phosphorus (n-type, $<100>$, concentration of $\sim 2 \times 10^{16} \mathrm{~cm}^{-3}$ ) ) doped wafers are used for synthesis of the Si-ncs. The wafers are adhered to the bottom of a glass container and immersed in $10 \mathrm{ml}$ water. The laser beam is focused onto a $1.5 \mathrm{~mm}$ diameter spot on the wafer surface by a lens with a focal length $25 \mathrm{~cm}$. The ablation process is continued for 2 hours at room temperature and ambient pressure. Following the ablation process the aqueous solution with produced Si-ncs is left to age in ambient conditions [12].

The Si-ncs used for fabrication of the blends were prepared at the same ablation conditions by using p-type doped silicon wafer. In order to obtain enough high Si-nc concentration the process was repeated several times. Si- 
ncs were aged for one month in water and then exposed to ambient condition in atmospheric air. It has to be noted that no specific protection from to the laboratory ambient light was conducted. Afterwards dried Si-ncs were introduced in P3HT polymer to produce the blend. A commercially available (ALDRICH) polymer P3HT was dissolved in chlorobenzene (12 $\mathrm{mg}$ of polymer in $10 \mathrm{~g}$ of chlorobenzene). The Si-ncs powder at $\sim 55$ wt. \% was mixed with polymer in order to make films on two types of substrates. Firstly, for low temperature PL investigations, the $300 \mathrm{~nm}$ thin films were spun on quartz substrates. Secondly, for a photoconductivity measurement the thin film at the same thickness was spun cast on a glass covered by interdigitated platinum contacts. The interdigitated contacts consisted of 20 fingers, with a length of $6 \mathrm{~mm}$ and a width of $200 \mu \mathrm{m}$, separated by $200 \mu \mathrm{m}$. At the same conditions another set of samples just with pure P3HT polymer was fabricated for comparison. For all cases the samples were dried at $140{ }^{\circ} \mathrm{C}$ for $30 \mathrm{~min}$ in vacuum.

The PL measurement of the colloids was performed at room temperature and ambient atmosphere. For PL measurements (Spectrofluorometer, Horiba Jobin Yvon) an excitation wavelength at $300 \mathrm{~nm}$ was used. For the temperature dependence of the PL, a HeCd laser (325 nm) has been used. The samples were placed in the cryostat with a varying temperature from 4 to $300 \mathrm{~K}$.

The conductivity and the photoconductivity measurements were conducted in ambient conditions. A voltage from a regulated DC power supply was applied. Resulting current was measured with an amperemeter (Sub Femtoamp, KEITHLEY 6430). For the photoconductivity measurement a white light of $1.5 \mathrm{AM}\left(100 \mathrm{~mW} / \mathrm{cm}^{2}\right)$ was used.

\section{Results and discussion}

\subsection{Si-ncs photoluminescence}

Similar to our previous investigations [11], as prepared Si-ncs did not show any visible PL at room temperature. Aging in water ( $\sim 1$ month) was required to obtain visible PL. The PL is stable and does not degrade under continuous exposure to the air or water [12]. Typical results on Sincs emission used in this study are depicted in Fig. 1. Image (a) shows Si-ncs prepared by laser ablation of n-type doped crystalline silicon wafer and image (b) p-type doped, respectively. Visible blue-room temperature PL is observed from both colloids under He:Cd laser excitation at $325 \mathrm{~nm}$. By naked eye, a stronger PL emission is noticed from ntype doped Si-ncs.

Corresponding PL spectra of colloidal solutions are shown in Fig. 1 (c). The solutions are excited at $300 \mathrm{~nm}$. At ambient conditions Si-ncs in colloidal solution show a stable blue-bands with maxima centered at $420 \mathrm{~nm}$. Compared to the Si-ncs made from p-type doped wafer (black line), the PL band for n-type doped (red symbols) is stronger more than 2 times. Even more, one can also observe that with an increase of the PL intensity slight blue-shift of the PL maxima position $(\sim 10 \mathrm{~nm})$ is recorded.
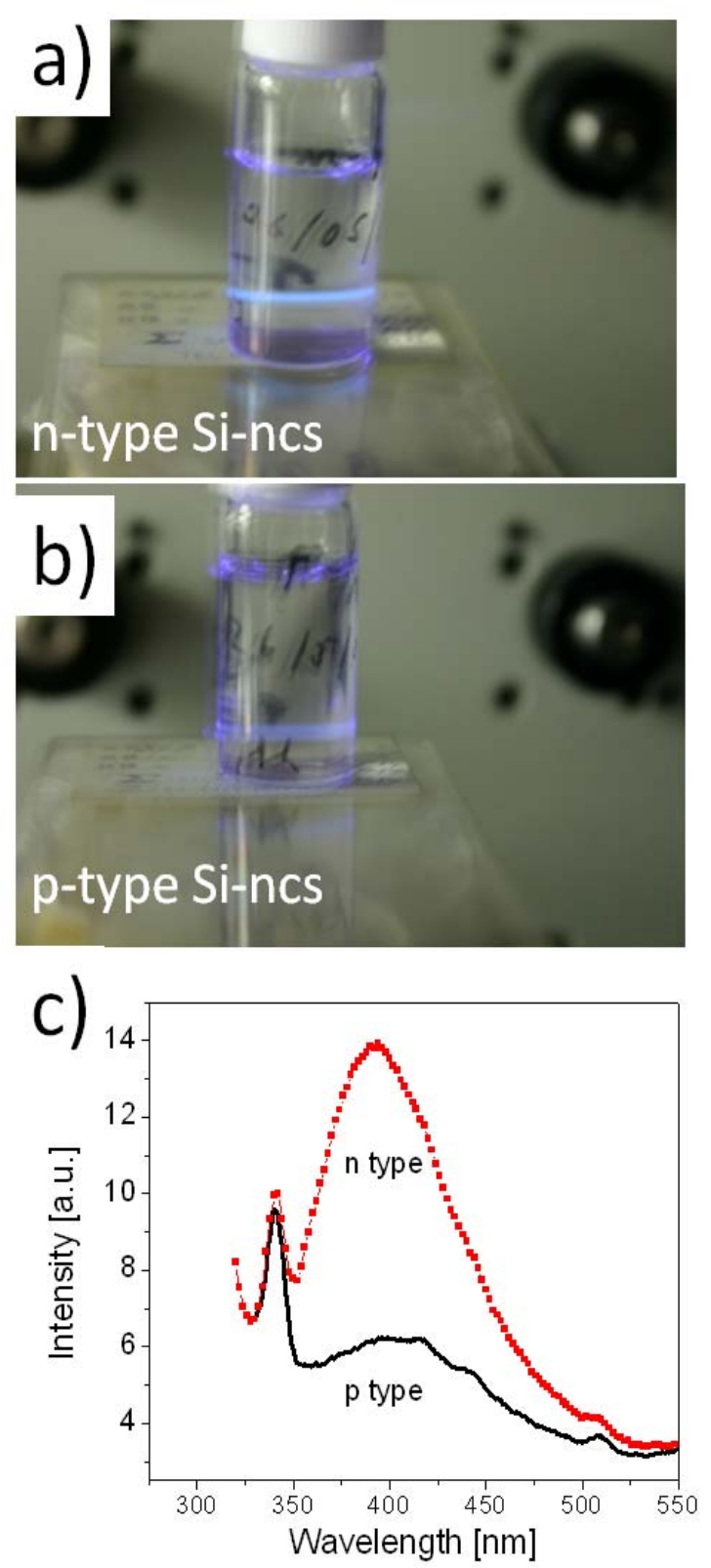

Figure 1. Photographs of blue-emission from silicon nanocrystals (Si-ncs) prepared by an excimer laser ablation in water and aged in water for 7 months. Photo (a) represents Si-ncs prepared from n-type doped and photo (b) of the ptype doped crystalline silicon wafer, respectively. Plot (c) shows corresponding PL spectra of doped Si-ncs taken at room temperature under excitation at $300 \mathrm{~nm}$.

Small peaks within the main PL band are also observed. Those are most likely related to the defects [12]. Laser ablation produced Si-ncs in water offers peculiar surface chemistry especially for the small sized Si-ncs $(<2 \mathrm{~nm})$ [12]. The oxide shell provides a natural and stable surface. Then the surface states are stabilized by the widening of the nanocrystals band gap induced by quantum confinement effect. Opening of energy band gap originates from electronic states in Si-ncs [15-17]. A smaller Si core size will cause an increase in the PL intensity due to quantum con- 
finement effects and more direct transitions. TEM analysis and Raman spectroscopy showed $[11,12]$ the presence of the Si-ncs with quantum confinement size $<3 \mathrm{~nm}$ that strongly supports quantum confinement contribution.

However, presence of a defect at the Si-oxide interface causes a decrease the PL intensity of the longer wavelength emission. The quantum confinement effect in nanocrystals results to a electron-hole pair radiative recombination in Si-ncs with different size distribution, which leads to a broadening of the PL spectra. In addition to that, a carrier trapping at silicon surface produces a stable states in the band-gap that contribute to radiative recombination as well $[5,12]$. It is well know that in case of Si-ncs, dopants are very often located at the surface of the nanocrystals and can contribute to the emission enhancement [18]. Since for n-type substrate a smaller surface recombination velocity has been reported compared to p-type Si wafers [19], similar effects on the nanocrystal surface could be expected and different surface passivation due to the dopant influences the PL intensity in doped nanocrystals. However, at this stage of the research the mechanism is not clear yet and more detailed studies are required.

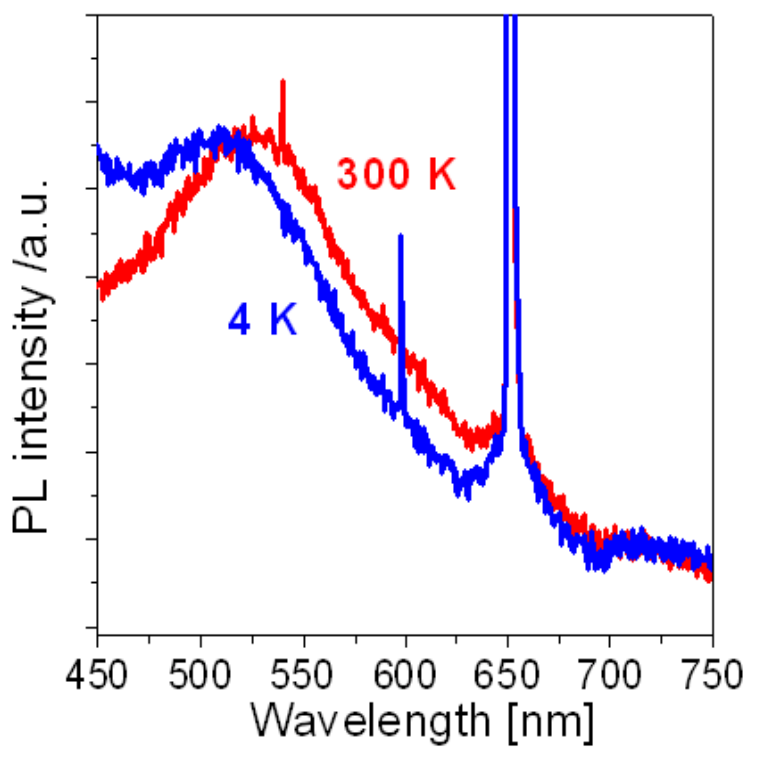

Figure 2. Normalized photoluminescence (PL) spectra of p-type doped Si-ncs exposed in air taken at $4 \mathrm{~K}$ (blue line) and room temperature $300 \mathrm{~K}$ (red line).

It is clear that for the photovoltaic application it is important and beneficial suppressed the radiative recombination. As a result an enhancement in photocurrent generation could be expected. Air-stable oxide surface layer may serve as a stable dielectric shell for further functionalization of nanocrystals and formation of new nanocomposite materials. Stable nanocrystal surface is an advantage in terms of degradation reduction when embedded in polymer matrix [20]. Since the laser ablation in water leads to the formation of an oxide layer on the Si-ncs through self-limiting process it is clear that for photovoltaic applications, a balance between the PL and thickness of surface oxide layer has to be find. In order to form conjugated P3HT polymer based blends larger amount of p-type doped, less luminescent Si-ncs powder was used. Corresponding PL spectra of dried Si-ncs powder and deposited on a quartz substrate are shown Fig. 2. It is well known that the temperature dependence of the PL can give an additional information about the presence of nanocrystals and might independently reveal the origins of nanocrystals emission [12]. The PL spectra of p-type doped Si-ncs taken at $4 \mathrm{~K}$ (blue line) and room temperature $(300 \mathrm{~K}$, red line) are demonstrated. The temperature-dependent PL spectra showed typical behavior for Si-ncs with quantum confinement effects and alike to those obtained by $\mathrm{Si}$ ions implantation in silicon dioxide based matrix [21]. The differences in the shape and peak position are observed due to the differences in synthesis route that we applied. The red-shift of the PL maxima results from the filling effect as the temperature increases [20].

\subsection{Excitation transfer energy}

The investigation of the temperature dependent PL of the blends prepared from air stable Si-ncs are summarized in Fig. 3. Fig. 3 (a) represents the PL spectra of p-type doped Si-ncs exposed in air (3 months) and blended with P3HT polymer. Blue line shows the PL spectra taken at $4 \mathrm{~K}$ and red line at room temperature $(300 \mathrm{~K})$. An excitation transfer energy from a P3HT polymer to the nanocrystal outcomes in a red-shift of the PL maxima as a function of the temperature. In the temperature range $4-300 \mathrm{~K}$ a monotonic red-shift is observed (blue symbols, Fig. 3(b)). In this temperature range the red-shift is of about $\sim 40 \mathrm{~nm}$.- Compared to the Si-ncs only (Fig. 2) the red-shift is more important. It has to be stressed that the PL of the pure polymer film is systematically blue-shifted (black squares, Fig. 3(b)).

The pronounced red-shift indicates that an electron interaction between the nanocrystal and polymer has been achieved. When the nanocrystal is capped by an organic layer (e. g. ligands) the excitation transfer is dominated by the Förster mechanism [22, 23]. This transfer is based on a dipole-dipole coupling process [24]. However, in our case during preparation process the Si-ncs surface is not covered by any surfactant (i.e. ligands), which limits potential dipole-dipole coupling. Contrary to that when the nanocrystal do not contains such groups and acceptor-donor are in a closer distance the excitation transfer is dominated by the charge exchange process (i.e. Dexter like energy transfer) [25]. In the Dexter mechanism, the spectral overlap is independent of the oscillator strength of the transitions and is efficient at very small distances $(<10 \AA)$ [26]. Then a close proximity of the donor and the acceptor overlaps of theirs wave functions [26]. In this particular case we assume that the donor and the acceptor are in a direct or at least in van der Waals contact. Therefore we expect that the Dexter energy transfer is most likely the responsible mechanism in excitation energy transfer [27]. However in our case the Sincs have their surface passivated by an oxide layer. This barrier increases the mean distance between the polymer 
and the nanocrystal and reduces of a Dexter transfer process [28]. Though, we believe that the transfer is enough efficient and leads to a decrease of the nanocrystals emission $<500 \mathrm{~nm}$ and red-shift of the blend PL spectra [29]. On the other hand, the localization of the wavefunction at the $\mathrm{Si}-\mathrm{ncs} / \mathrm{SiO}_{2}$ interface [30] increases the overlap with polymer and might not only favorites the Dexter energy like transfer but also overall transport of photogenerated carriers [29]. It has to be noted that the Dexter transfer of energy in other type of nanocrystal/ P3HT blends has been already observed and reported [31].
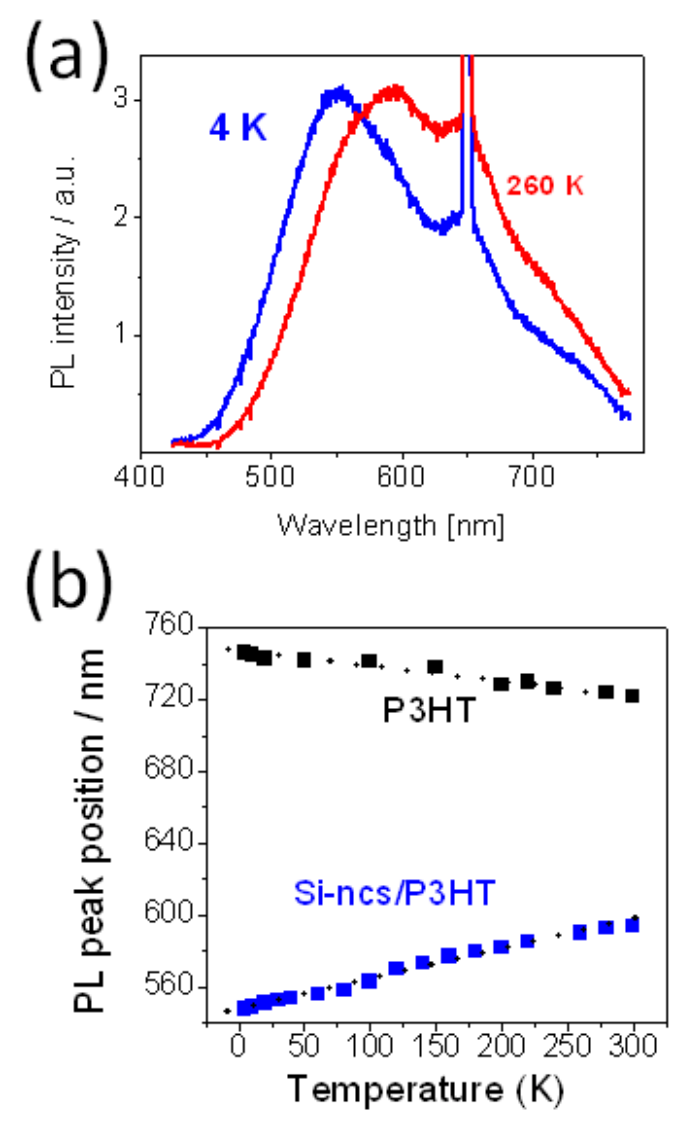

Figure 3. (a) Photoluminescence (PL) spectra of p-type doped Si-ncs exposed in air and blended in P3HT polymer are plotted. Blue line represents PL spectra taken at $4 \mathrm{~K}$ and red line at room temperature $(300 \mathrm{~K})$, respectively. (b) Summary of the PL peak position (blue symbols) as a function of the temperature is shown. Peak position of the pure P3HT polymer film only is shown for comparison (black symbols).

\subsection{Bulk-heterojunction}

Normalized optical transmission spectra of the p-type doped Si-ncs blended in P3HT polymer (blue line) and pure P3HT polymer film (squares) are depicted in Fig. 4. Due to an enhanced polymer absorption curves show similar transmission pattern. No significant shift of the minima ( $560 \mathrm{~nm}$ ).indicates that no major changes in polymer interchain association and low nanocrystals aggregation was achieved. The PL emission of the pure polymer film is decreased by factor of $\sim 2$ when the Si-ncs are introduced. Quenching in PL promotes a photo-induced charge transfer from the polymer to nanocrystal. One could speculate that such a blend would be an useful composite material to fabricate photosensitive devices as solar cells or photodetectors.

Furthermore, the photo-transport properties of the blend are investigated. Surprisingly, a blend based even on oxidized and room-temperature blue luminescent Si-ncs shows photoconductivity properties. Figure 5 (a).represents current-voltage (I-V) characteristics for such Si-ncs blended with P3HT polymer and spin-cast on platinum interdigitated contact. The blend characteristics in dark (black lines) and under AM1.5 irradiation (red lines) are presented after annealing at temperature $140{ }^{\circ} \mathrm{C}$. An improvement in darkand photo-conductivity response is recorded. It has to be noted that at chosen concentration $(\sim 55 \mathrm{wt} . \%)$ allows formation of percolation trajectories for photogenerated carriers. The ratio between photo- and dark-conductivity is reaching value of about $\sim 1.7$. As the Si-ncs concentration is further increased the ratio decreases due to the perturbation of the polymer chains.

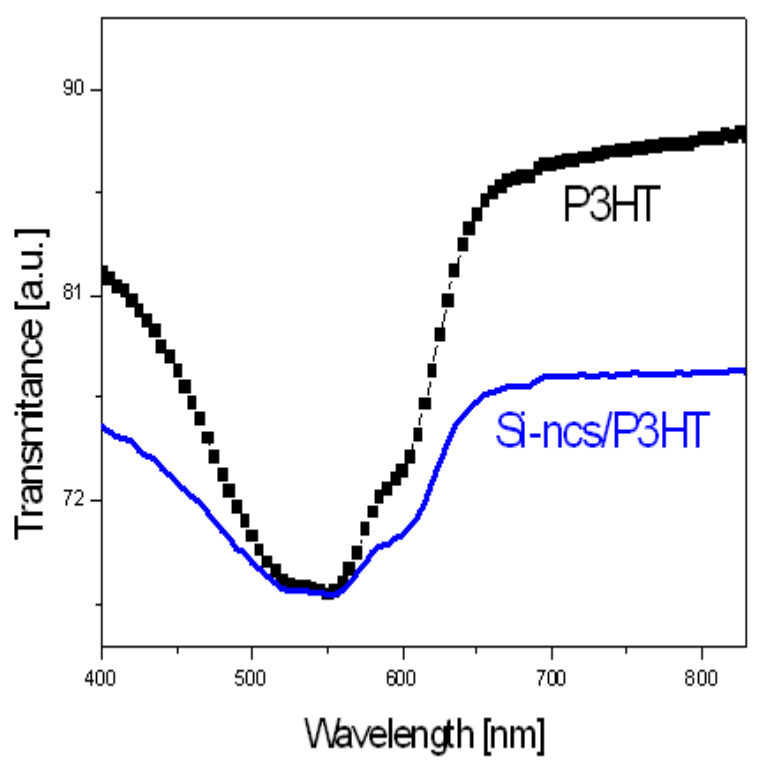

Figure 4. Transmission spectra of p-type doped Si-ncs blended in P3HT polymer (blue line) and pure P3HT polymer film (squares) are shown.

Similar to the Si-ncs prepared by electrochemical etching, it is believed that electronic interaction of Si-ncs with P3HT polymer leads to the bulk-heterojunction formation [20]. Proposed energy level diagram of Si-ncs and conjugated P3HT polymer based blend is given in Fig. 5(b). Value for an ionization energy of Si-ncs is taken from the PL and the absorption measurements with respect to the characteristics of the bulk silicon ( $\mathrm{Si}$ ) [12]. The highest occupied molecular orbital (HOMO) is at $(\sim 5 \mathrm{eV})$ and low- 
est unoccupied molecular orbital (LUMO) level is at $\sim 2.9 \mathrm{eV}$. Since the Si-ncs having a large optical band gap $(\sim 2.9 \mathrm{eV})$ and work function being around $-4.1 \mathrm{eV}$ alignment with polymer is assured. Different electron affinity and ionization potential between the nanocrystal and polymer provide a driving force for dissociating the excitons. An electron is swept into the nanocrystal while the hole goes to the polymer [14]. As a result an increase in photocurrent generation is observed when the blends are irradiated by AM1.5.
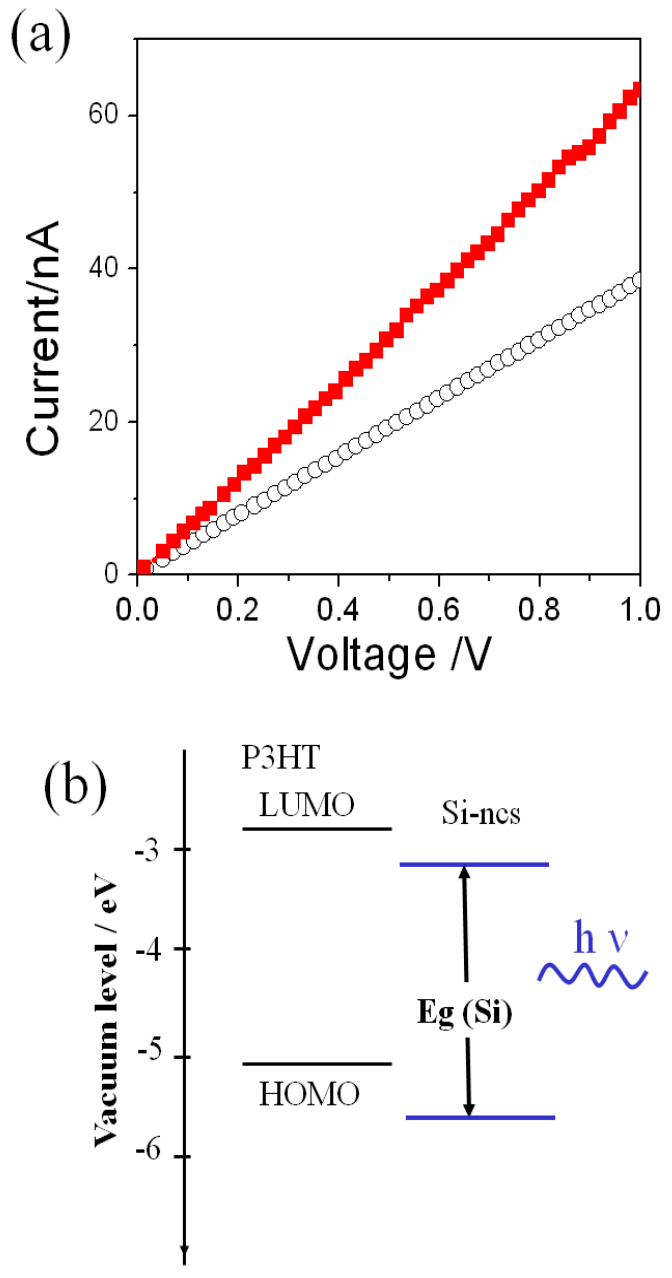

Figure 5. (a) Current-voltage (I-V) characteristics for blue silicon nanocrystals (Si-ncs) blended with P3HT polymer and spin-cast on platinum interdigitated contact are shown. The dark (black lines) and under illumination (red lines) at AM1.5 I-V are presented after annealing at temperature $140{ }^{\circ} \mathrm{C}$. (b) Proposed energy level diagram of Si-ncs/ P3HT polymer based blends. Value for position of energy levels of Si-ncs is taken from the photoluminescence and the absorption measurements [12].

The results indicate that conjugated P3HT polymer blended with an oxide surface layer of Si-ncs owing a quantum confinement effects may provide a prospective for development of low-cost photovoltaic devices. Since the Si-ncs prepared by laser ablation in water might naturally contain an oxide layer, which can hinder the overall transport properties, the thickness should be reduced. It is be- lieved that this could be over came by preparation of the Si-ncs in the less oxidizing liquid medium or polymer solvent (i.e. chlorbenzene).

\section{Conclusions}

In conclusion, the fabrication of silicon nanocrystals (Si-ncs) from p- and n-type doped crystalline wafer by laser processing has been presented. Cost-effective nanosecond $\mathrm{KrF}$-excimer laser processing in water offers fabrication conditions for Si-ncs with stable blue emission at room temperature. The self-limiting oxidation in water and the oxide shell provide high quality surface passivation that results an efficient electron-hole recombination. Those states are stabilized by the widening of the nanocrystals band gap induced by quantum confinement, which is more pronounced for n-type doped Si-ncs.

Furthermore, the blending of the less luminescent $\mathrm{p}$ type doped Si-ncs with P3HT polymer is demonstrated. Fabrication of photosensitive blends has been successfully achieved. Contrary to the PL blue-shift of pure polymer films, an excitation transfer energy from the polymer to the nanocrystals results in PL red-shift of about $40 \mathrm{~nm}$ in the temperature range of 4-300 K.. Due to the formation of the bulk-heterojunction at Si-ncs/P3HT interface, the photoconductivity generation under AM1.5 irradiation has been observed.

\section{Acknowledgments}

This work was also partially supported by a NEDO project.

\section{References}

[1] Y. Xia and N. J. Halas, MRS Bull., 30 (2005) 338.

[2] M. C. Daniel and D. Astruc, Chem. Rev., 104 (2004) 293.

[3] F. Mafune' and T. Kondow, Chem. Phys. Lett., 383 (2004) 343.

[4] S.I. Dolgaev, A.V. Simakin, V.V. Voronov, G.A. Shafeev, and F. Bozon-Verduraz, Appl.Surf.Sci., 186 (2002) 546.

[5] M. V. Wolkin, J. Jorne, P. M. Fauchet, G. Allan, and C. Delerue, Phys. Rev. Lett. 82, (1999) 197.

[6] L. Pavesi, L. D. Negro, L. Mazzoleni, G. Franzo, and F. Priolo, Nature (London) 408 (2000) 440.

[7] R. J. Ellingson et al., Nano Lett. 5, (2005) 865.

[8] M. Sykora et al., Phys. Rev. Lett. 100, (2008) 067401.

[9] V. Švrček, T. Sasaki, Y. Shimizu, and N. Koshizaki, Appl. Phys. Lett 89, (2006) 213113(1-3).

[10] S. Yang, W. Cai, H. Zeng, and Z. Li, J. of Appl. Phys. 104 (2008) 023516 (1-5),

[11] V. Švrcek, T. Sasaki, R.Katoh, T. Shimizu and N. Koshizaki, Appl. Phys. B 94 (2009) 133.

[12] V. Švrček, D.Mariotti, and M. Kondo, Optics Express 17 (2009) 520

[13] V. Švrček, T. Sasaki, Y. Shimizu and N. Koshizaki, J. Appl. Phys., 103 (2008) 023101

[14] V. Švrček, H. Fujiwara, M. Kondo, Appl. Phys. Lett. 92 (2008) 143301. 
[15] X.L. Zheng, W. Wang, H.C. Chen, Appl. Phys. Lett. 60 (1992) 986.

[16] S. Sinha, S. Banerjee, B.M. Arora, Phys. Rev. B 49 (1994) 5706.

[17] G. Ledoux, O. Guillois, D. Porterat, C. Reynaud, F. Huisken, B. Kohn and V. Paillard, Phys. Rev. B 62 (2000) 15942.

[18] M. Fujii, A. Mimura and S. Hayashi, Phys. Rev. Lett. 89 (2002) 296805.

[19] E. Yablonovitch, D. L. Allara, C $>$ C. Chang, T. Gmitter, T. B. Bright, Phys. Rev. Lett. 57, (1986) 249.

[20] V. Švrček, H. Fujiwara, M. Kondo, Solar Energy Materials \& Solar Cells 93 (2009) 774.

[21] M. L. Brongersma, P. G. Kik, A. Polman, K. S. Min, and H. A. Alwater, Appl. Phys. Lett. 76 (2000) 351.

[22] Th. Förster, Ann. Phys. (N.Y.) 2, (1948) 55.

[23] Th. Förster, Discuss. Farraday Soc. 27, (1959) 7.

[24] V. L. Colvin, M. C. Schlamp, and A. P. Alivisatos, Nature 370 (1994) 354.
[25] N. Tessler, V. Medvedev, M. Kazes, S. Kan, U. Banin Science 295 (2002) 1506 .

[26] L. Bakueva, S. Musikhin, M. A. Hines, T. W. F. Chang, M. Tzolov, G.D. Scholes, E.H. Sargent, Appl. Phys. Lett. 82 (2003) 2895.

[27] A.A.R.Neves, A. Camposeo, R. Cingolani, D. Pisignano, Adv. Funct. Mater. 18 (2008) 751.

[28] N. Greenham, X. Peng, A. Alivisatos, Phys. Rev. B, 54 (1996) 17628.

[29] P.I. Archer, S.A. Santangelo and D.R. Gamelin, J. Am. Chem. Soc. 129 (2007) 9808.

[30] N. Daldosso, M. Luppi, S. Ossicini, E. Degoli, R. Magri, G. Dalba, P. Fornasini, R. Grisenti, F. Rocca, L. Pavesi, S. Boninelli, F. Priolo, C. Spinella, and F. Iacona, Phys. Rev. B 68, (2003) 085327.

[31] C.-M Yang, C.-H., Wu, H.-H Liao, K.-Y. Lai, H.-P. Cheng, S.-F Horng, H.-F., Meng, and J.-T. Shy, Appl. Phys. Lett. 90, (2007) 133509.

(Received: June 26, 2009, Accepted: February 27, 2010) 